\title{
BERNOULLI CONVOLUTIONS AND DIFFERENTIABLE FUNCTIONS
}

BY

R. KAUFMAN $\left({ }^{1}\right)$

ABSTRACT. Bernoulli convolutions, similar in structure to convolutions with a constant ratio, are considered in relation to differentiable transformations. A space of functions on the Cantor set leads to highly singular measures that nevertheless resemble absolutely continuous measures sufficiently to control their Fourier-Stieltjes transforms.

To each number $\theta$ in $(0,1)$ there corresponds a measure $\mu$ whose FourierStieltjes transform $\hat{\mu}$ is defined by $\hat{\mu}(u)=\Pi_{1}^{\infty} \cos \left(u \theta^{n}\right)$. Moreover, $\hat{\mu}$ vanishes at infinity unless $\theta^{-1}$ is a PV number and $\theta \neq 1 / 2[6$, pp. 36-41], $[8$, pp. 147152]. The measure $\mu$ has an interesting property apparently not observed until now, and this property is shared by measures close to $\mu$ in structure. Operating in a certain space of functions on a Cantor set, we can produce highly singular measures with the same property.

Let $S=\left(\theta_{n}\right)_{1}^{\infty}$ be a sequence of numbers in $(0,1)$, and let $\theta=\lim \theta_{n}, 0<$ $<\theta<1$, fulfill the condition for the vanishing of $\hat{\mu}(\infty)$ mentioned before. We define $\lambda$ by the formula $\hat{\lambda}(u)=\Pi_{1}^{\infty} \cos \left(u \theta_{1} \cdots \theta_{n}\right)$, observing that $\lambda$ is carried by the set $\Sigma(S)$ of all infinite sums $\Sigma \pm \theta_{1} \cdots \theta_{n}$.

THEOREM 1. The measure $\lambda$ has the property

$$
\lim \int \exp i u \varphi(t) \cdot \lambda(d t)=0
$$

for every $\varphi$ in $C^{1}(-\infty, \infty)$ with $\varphi^{\prime}>0$ everywhere.

THEOREM 2. For each Hausdorff measure-function $h$, there is a probability measure $\lambda_{1}$ with property $\left(R_{1}\right)$, whose support has $h$-measure 0 and is contained in $\Sigma(S)$.

1. The next lemma contains a substantial part of the analysis in the theorems. To each $A>1$ we denote by $\hat{\lambda}_{A}(u)$ the partial product in $\hat{\lambda}(u)$, extended over indices $n$ for which $\left|\theta_{1} \cdots \theta_{n} u\right|<A$.

Received by the editors July 18, 1974.

AMS (MOS) subject classifications (1970). Primary 42A72; Secondary 46E15, 28A10. Key words and phrases. Bernoulli convolution, $M_{0}$-set.

(1)Alfred P. Sloan Fellow.

Copyright $\odot$ 1976, American Mathematical Society 
LEMmA. To each $\epsilon>0$ there is an $A$ so large that $\left|\hat{\lambda}_{A}(u)\right|<\epsilon$ when $|u|>A$.

To prove this we choose $A$ so that $|\hat{\mu}(u)|<\epsilon$ when $|u|>A$. For large $u$ $\hat{\lambda}_{A}(u)=\Pi_{1}^{\infty} \cos \left(v \varphi_{1} \cdots \varphi_{n}\right)$, wherein $A \leqslant v<A \theta^{-2}$ and the numbers $\varphi_{1}, \ldots$, $\varphi_{n}, \ldots$ belong to the tail of the sequence $S$-in fact they belong to $\left(\theta_{n}\right)_{p}^{\infty}$, with $\theta_{1} \cdots \theta_{p}|u|<A$. The infinite product is then uniformly close to $\hat{\mu}(v)$, for large $u$, and this proves the lemma.

Instead of giving the proof of Theorem 1 immediately, we introduce some machinery leading to Theorem 3, a stronger result. Let $C$ be the Cantor set of sequences $x=\left(x_{\kappa}\right)_{1}^{\infty}, x_{\kappa}=-1,+1$. $C$ is a topological group and the Haar measure $\sigma$ is the customary product measure. The distance $d\left(x, x^{\prime}\right)=2^{-N}$ if $x=$ $x_{1}^{\prime}, \ldots, x_{N}=x_{N}^{\prime}$ but $x_{N+1} \neq x_{N+1}^{\prime}$. The modulus of continuity $\omega_{k}(f)$ of a mapping $f$ of $C$ into a metric space-in particular into $C$ itself-is given by

$$
\omega_{\kappa}(f, x)=\sup d\left(f\left(x^{\prime}\right), f(x)\right): d\left(x, x^{\prime}\right) \leqslant 2^{-\kappa} .
$$

The space $\lambda_{1}^{0}$ is composed of continuous mappings of $C$ into itself such that $2^{\kappa} \omega_{\kappa}(f) \rightarrow 0$ in $\sigma$-measure, while $\lambda_{1}^{p}(1 \leqslant p<\infty)$ is defined by the same relation in $L^{p}(\sigma)$. These spaces are complete metric groups.

$C$ is mapped onto $\Sigma(S)$ by the transformation $Y(x)=\Sigma \theta_{1} \cdots \theta_{\kappa} x_{\kappa}$ and this also maps the measure $\sigma$ onto $\lambda$, i.e. $\lambda=\sigma \circ Y^{-1}$ as set-functions.

THEOREM 3. Let I denote the identity map of $C$, let $f$ belong to $\lambda_{1}^{0}$, and let $I+f$ denote a sum in the group $C$. Then $Y_{1}=Y_{0}(I+f)$ transforms $\sigma$ onto a measure $\lambda_{1}$ with property $\left(R_{1}\right)$.

Proof. For large $u$ we define $n(u)=n$ to be the least integer such that $\left|\theta_{1} \cdots \theta_{n} u\right|<A$ ( $A$ is fixed until the end of proof). Then we divide $C$ into cylinders $C_{j}\left(1 \leqslant j \leqslant 2^{n}\right)$ of $\sigma$-measure $2^{-n}$. Of course these are just cosets of the subgroup $x_{1}=\cdots=x_{n}=+1$. Now $\omega_{n}(f)$ is constant on each $C_{j}$-let its value be $2^{-n_{j}}$. On $C_{j}$ we can write

$$
Y(x+f(x))=Y\left(x+\bar{x}_{j}\right)+O\left(\theta_{1} \cdots \theta_{n_{j}}\right),
$$

for some element $\bar{x}_{j}$ of $C$. Now $n_{j}-n \rightarrow+\infty$ except on sets $C_{j}$ whose total $\sigma$ measure tends to 0 , and when $n_{j}-n$ is large, then $|u| \theta_{1} \cdots \theta_{n_{j}}$ is correspondingly small. Therefore we can focus on the estimation of $\exp \operatorname{iu\varphi } \bullet Y\left(x+\bar{x}_{j}\right)$, and observe that uniform continuity of $\varphi^{\prime}$ gives

$$
u \varphi \circ Y\left(x+\bar{x}_{j}\right)=u \varphi^{\prime}\left(\bar{y}_{j}\right) \cdot Y\left(x+\bar{x}_{j}\right)+\bar{z}_{j}+o(1),
$$

where $\bar{y}_{j}$ belongs to $\Sigma(S)$, whence $\varphi^{\prime}\left(\bar{y}_{j}\right) \geqslant \eta>0$. Thus the integral over $C_{j}$ has absolute value $\left\langle o\left(2^{-n}\right)+2^{-n}\left|\hat{\lambda}_{A}(v)\right|\right.$, with $|v| \geqslant \eta|u|$. By the lemma we can make this $<\epsilon 2^{-n}$ by increasing $A$ and $|u|$, and this proves the theorem. 
THEOREM 2'. For each Hausdorff measure-function $h, I+f$ maps $C$ onto a set of h-measure 0 in $C$, except for a subset of first category of $\lambda_{1}^{p}$.

In the proof we use operators $T_{\kappa}$, defined by

$$
T_{\kappa} f(x)=f\left(x_{1}, \ldots, x_{\kappa}, 1,1, \ldots\right) .
$$

Clearly $\omega_{N}\left(T_{\kappa} f-f\right) \leqslant \omega_{N}(f)$, and of course

$$
\omega_{N}\left(T_{\kappa} f-f\right) \leqslant \sup d\left(T_{\kappa} f(x), f(x)\right) .
$$

Therefore $T_{\kappa} f \rightarrow f$ uniformly as $\kappa \rightarrow+\infty$ and moreover

$$
\sup _{N} 2^{N}\left\|\omega_{N}\left(T_{k} f-f\right)\right\|_{p} \rightarrow 0 .
$$

Thus $\lambda_{1}^{p}$ contains a dense subgroup of functions $f$ with a finite range. Suppose, for definiteness, that $f$ is constant on sets of diameter $2^{-r}$ and let $N>r, N=$ $N_{1}<M_{1}<\cdots<N_{q}<M_{q}<\cdots, 1 \leqslant q \leqslant 2^{N}$. We divide $C$ into the standard sets $C_{q}$ of measure $2^{-N}$ and define

$$
\psi(x)=T_{N}(x)+T_{M}(x) \text { on } C_{q}\left(N=N_{q}, M=M_{q}\right) .
$$

In estimating $\left\|\omega_{\kappa}(\psi)\right\|_{p}$ we first consider $\kappa=1,2, \ldots, N_{1}$. Now $d(\psi, 1) \leqslant$ $2^{-N}$ everywhere and $d(\psi, 1) \leqslant 2^{-N} 2$ except on $C_{1}$. But $C_{1}$ meets exactly one of the cylinders of index $k$, so $\left\|\omega_{\kappa} x\right\|_{p} \leqslant 2^{-N_{2}} 2^{-\kappa / p}+2^{-N_{2}}$. This is small in comparison with $2^{-\kappa}$, provided $N$ and $N_{2}-N_{1}$ are large. When $N_{1} \leqslant \kappa \leqslant M_{1}$ we find $\left\|\omega_{\kappa}(\psi)\right\|_{p} \leqslant 2^{-\kappa} 2^{-N / p}+2^{-N_{2}}$, and this can be made small enough by increasing $N$ and $N_{2}-M_{1}$. When $\kappa \geqslant M_{1}$, the argument remains the same, except that $\omega_{\kappa}(\psi)=0$ on $C_{1}$, etc. Thus $\psi$ has a small norm in $\lambda_{1}^{p}$, for appropriate choices of $N, N_{1}, M_{1}, \ldots$.

It remains to investigate the mapping properties of $f(x)+x+\psi(x)$. On $C_{q}$, this equals $f(x)+x+T_{M}(x)+T_{N}(x)$. Using the fact that $f$ is constant on sets of diameter $2^{-r}$, and $N=N_{q}>r$, we see that $C_{q}$ is mapped into at most $2^{N}$ sets of diameter $2^{-M}$. By making the sum $\Sigma 2^{N} q \cdot h\left(2^{-M_{q}}\right)<\eta$ (say), we obtain a function $f+\psi$, close to $f$ in $\lambda_{1}^{p}$, such that $I+f+\psi$ transforms $C$ into a union of sets $B_{s}$, with $\Sigma h\left(\operatorname{diam} B_{s}\right)<\eta$. Moreover, this remains true for all $f^{*}$ in a neighborhood $W^{*}$ of $f+\psi$, because the metric in $\lambda_{1}^{p}$ is stronger than the uniform metric. Taking a sequence $\eta_{r} \rightarrow 0$, we obtain a dense $G_{\delta}$-set in $\lambda_{1}^{p}$ with the property that $(I+f) C$ has $h$-measure 0 .

Theorem $2^{\prime}$ leads directly to Theorem 2 because $Y$ is uniformly continuous: to each $h$ there is an $h_{1}$ so that $h$-mes $Y(F) \leqslant h_{1}$-mes $F$ for every subset $F$ of $C$.

2. In the definition of property $\left(R_{1}\right)$, the continuity of $\varphi^{\prime}$ is essential. If, for example, we allow all absolutely continuous functions $\varphi$, with $1<\varphi^{\prime}<2$ a.e., then the corresponding measures would necessarily be absolutely continuous [6]. Therefore it is interesting to state a theorem in which $\varphi^{\prime}$ need not be continuous, 
nor even locally integrable. For this kind of theorem we take $\theta_{n} \equiv 2$; although this can be avoided, the complications are great. Compare [1], [7].

THEOREM 4. Suppose that $\varphi(t)$ is defined on $[-1,1]$ and is differentiable almost everywhere with derivative $\varphi^{\prime} \neq 0$. Let $f \in \lambda_{1}^{0}$. Then for almost all $y$ in $C$, the mapping $H_{y}: H_{y}(x)=\varphi \circ Y(x+y+f(x))$ transforms the measure $\sigma$ onto a measure $\lambda_{1}$ such that $\hat{\lambda}_{1}(\infty)=0$.

The proof depends on Marcinkiewicz' variant of Lusin's theorem [8, pp. 73-77]: to each $\epsilon>0$ there is a function $\varphi_{*}$ of class $C^{1}[-1,1]$, such that $m\left\{\varphi \neq \varphi_{*}\right\}<\epsilon$. Now $\varphi^{\prime}=\varphi_{*}^{\prime}$ a.e. on $\left\{\varphi=\varphi_{*}\right\}$ so there is a closed set, of measure $>2-\epsilon$, on which $\varphi_{*}^{\prime} \neq 0$ and $\varphi=\varphi_{*}$. By parabolic interpolation [8] we can adjust $\varphi_{*}$ off the closed set so that it remains in $C^{1}[-1,1]$ and $\varphi_{*}^{\prime} \neq 0$ except on a countable set.

We claim that

$$
\lim \int \exp \operatorname{iu} \varphi_{*} \circ Y(x+y+f(x)) \sigma(d x)=0
$$

for every $y$ in $C$. Indeed, if $J \subseteq(-1,1)$ is an interval on which $\varphi_{*}>0$ or $\varphi_{*}<$ 0 , then $\varphi_{*}$ can be extended from $J$ to a function $\Phi$ of class $C^{1}(-\infty, \infty)$, with $\Phi^{\prime}$ $>0$ or $\Phi^{\prime}<0$ everywhere. By Theorem 2 and a known theorem [8, p. 145], $\lim \int_{J}(\cdot)=0$. Since $\varphi_{*}^{\prime}=0$ only on a countable closed set, this is sufficient to establish our claim.

Now the $\sigma$-measure of the $x$-set

$$
\varphi \circ Y(x+y+f(x)) \neq \varphi_{*} \circ Y(x+y+f(x))
$$

is a Lebesgue-measurable function of $y$, whose integral over $C$ is $<\epsilon$. So the $\sigma$ measure is $<\epsilon^{1 / 2}$, except for a $y$-set of measure $<\epsilon^{1 / 2}$. Under this bound on the $\sigma$-measure

$$
\lim \sup \left|\int \exp i \mu \varphi \circ Y(x+y+f(x)) \sigma(d x)\right|<\epsilon^{1 / 2} .
$$

Since $\epsilon$ was arbitrary, the limit is 0 for almost all $y$. In these arguments we used the observation that $\lambda$, the distribution of the function $Y$, is $d t / 2$ on $(-1,1)$, and combined this with Fubini's theorem.

Differentiability of $\varphi^{\prime}$ is not the weakest condition for Marcinkiewicz' interpolation process $[8$, p. 228] and the theorems cited lead to a stronger version of Theorem 4. If we allow $\lambda$ to be singular, for example by taking $\theta<1 / 2$, then $\varphi^{\prime}$ must exist a.e. for $\lambda$. Marcinkiewicz' theorem becomes quite technical in this situation.

3. In this final section we suppose $\theta_{n}=\theta<1 / 2$ for all $n$ and write $\Sigma(\theta)$ for the support of the measure $\mu$. If $M$ is an infinite set of positive integers, then 
$\Sigma(\theta, M)$ stands for the set of sums $\Sigma x_{n} \theta^{n}$ in which $x_{n}=-1,+1$ and $x_{n}=+1$ for $n \notin M$. Then $\Sigma(\theta, M)$ carries a measure $\lambda$ whose Fourier-Stieltjes transform is

$$
\prod_{M} \cos u \theta^{n} \times \prod_{M^{\prime}} \exp -i u \theta^{n}
$$

As we observed earlier $\Sigma(\theta)$ admits decompositions into $2^{n}$ cylinders; their diameter is exactly $2 \theta^{n+1}(1-\theta)^{-1}$ and their distances at least $\left[1-\theta(1-\theta)^{-1}\right]$. $2 \theta^{n}$. When $M^{\prime}$ contains segments of unbounded length, then some function $\varphi$ of class $C^{1}(-\infty, \infty)$, with $\varphi^{\prime}>0$, transforms $\Sigma(\theta, M)$ onto a set of uniqueness $[3,2 \mathrm{VII}]$. Incidentally, this is also true when $\theta=1 / 2$, but then $\Sigma(1 / 2, M)$ is a set of uniqueness as soon as $M^{\prime}$ is infinite. Let us say that $M$ is deficient if $M^{\prime}$ contains segments of unbounded length.

THEOREM 5. Let $h$ be a positive function on $(0,4)$ and $h(0+)=0$. Then for a certain deficient sequence $M$ the product measure $\lambda$ on $\Sigma(\theta, M)$ has this property:

$$
\lim \int \exp -i u \varphi(t) \cdot \lambda(d t)=0
$$

whenever $\varphi \in C^{1}[-2,2], \varphi^{\prime}>0$ everywhere, and $\left|\varphi^{\prime}(a)-\varphi^{\prime}(b)\right| \leqslant h(|b-a|)$ for all $a, b$ in $[-2,2]$.

Sets with the two properties claimed for $\Sigma(\theta, M)$ were first constructed in [4], by a complex process; the examples given below are essentially variants of Theorem 1.

By the hypothesis on $h$, there is a function $\Gamma(u)>0$, defined and increasing without limit for $u>1$, such that $\Gamma(u) h\left(u^{-1} \Gamma(u)\right) \rightarrow 0$. It is convenient to set $\Gamma(u)=\Gamma(|u|)$ if $u<-1$.

Now we are led to define the infinite product $\lambda_{*}(u)=\Pi \cos u \theta^{n}: \theta^{n}|u|<$ $\Gamma(u)$. We shall prove that if $\lim \lambda_{*}=0$, then $\lambda$ has the property claimed in Theorem 5. First we choose $\delta>0$ so that $\delta<\varphi^{\prime}<\delta^{-1}$ on $[-2,2]$, and define $n=$ $n(u)$ to be the largest integer such that $|u| \theta^{n} \geqslant \delta^{-1} \Gamma\left(\delta^{-1} u\right)$. Now $\Sigma(\theta)$ is covered by cylinders of order $n$, of length $<\theta^{n+1}(1-\theta)^{-1} \leqslant 2 \delta u^{-1} \Gamma\left(\delta^{-1} u\right)=L$, say. Here $u L h(L) \rightarrow 0$ by the characteristic property of $\Gamma$. Thus $\varphi$ is represented on each cylinder by a linear function $a t+b$, with an error $o\left(u^{-1}\right)$, and $\delta<a<$ $\delta^{-1}$. The integral of exp - iuat over a cylinder has magnitude $2^{-n} \Pi_{n}^{\infty}\left|\cos u \theta^{m}\right|$. The latter product is no larger than $\left|\lambda_{*}(a u)\right|$, because the inequality $\theta^{m}|a u|<$ $\Gamma(a u)$ leads to $\theta^{m}|u|<\delta^{-1} \Gamma\left(\delta^{-1} u\right)$, so $m \geqslant n$. Since $\lambda_{*}$ vanishes at infinity, $\lim \int \exp -i u \varphi \cdot d \sigma=0$.

To finish the proof of Theorem 5, we exhibit a deficient set $M$ such that $\lambda_{*}$ vanishes at infinity. The complement $M^{\prime}$ will be a union of segments $p_{r} \leqslant n$ $\leqslant p_{r}+r$ with $p_{1}>1$ and $p_{r+1}>3 r+p_{r}(r=1,2,3, \ldots)$. The characteristic 
property of $\theta$, that $\theta^{-1} \notin P V$, is equivalent to $\Pi_{1}^{\infty} \cos u \theta^{-n}=0$ for every $u \neq 0$ (see the references in the introduction). By Dini's theorem on monotone sequences, the convergence to 0 is uniform on every compact subset of the positive line. Thus there are numbers $Q=Q(r)$ so that $\Pi_{1}^{Q}\left|\cos u \theta^{-n}\right|<r^{-1}$ for $\theta \leqslant u \leqslant$ $\theta^{-r}$. Now let $p_{1}, p_{2}, \ldots, p_{r}$ be defined so that for $r=1,2,3, \ldots$

$$
p_{r}>p_{r-1}+r+Q(2 r) \text { and } \Gamma\left(\theta^{\left.-p_{r}\right)}>\theta^{-Q(2 r)} \theta^{-2 r}\right. \text {. }
$$

In proving that $\lambda_{*}$ vanishes at infinity we define $s=s(u)$ by the inequality $\theta<\theta^{s} u \leqslant 1(u>1)$ and examine two possible inequalities for $s$ in relation to $M^{\prime}:$ either $p_{r}+2 r \leqslant s<p_{r+1}$ for a certain $r$, or $p_{r}<s<p_{r}+2 r$. One of these must obtain, as soon as $s>p_{1}$. In the first case $\lambda_{*}(u)$ contains all factors $\cos u \theta^{n}$ in which $p+r<n<s$ and $u \theta^{n} \leqslant \Gamma(u)$. In particular, this extends to all $n$ such that $1 \leqslant s-n \leqslant r-1$ and $\theta^{n-s} \leqslant \Gamma(u)$. In different terms $\lambda_{*}(u)$ contains all factors $\cos u \theta^{s} \cdot \theta^{-q}$ wherein $1 \leqslant q<r-1, \theta^{-q} \leqslant \Gamma(u)$. Now $\theta<u \theta^{s}$ $\leqslant 1$, while $r$ and $\Gamma(u)$ increase without bound, so this situation is covered by Dini's theorem.

In the case $p_{r}<s<p_{r}+2 r, \lambda_{*}(u)$ contains all factors wherein $p_{r-1}+r$ $\leqslant n<p_{r}$ and $u \theta^{n}<\Gamma(u)$. Now $u \theta^{n} \leqslant \theta^{n-s}$, and $\Gamma(u) \geqslant \Gamma\left(\theta^{-1} \theta^{-s}\right)>$ $\theta^{-Q(2 r)} \theta^{-2 r}$. Hence the inequalities on $n$ are fulfilled if $p_{r}-Q(2 r) \leqslant n<p_{r}$.

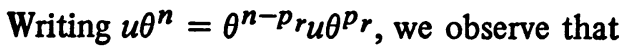

$$
\left|\lambda_{*}(u)\right| \leqslant \prod_{1}^{Q(2 r)}\left|\cos v \theta^{n}\right|, \quad \text { with } \theta \leqslant v \leqslant \theta^{-2 r} \text {, }
$$

so $\left|\lambda_{*}(u)\right|<r^{-1}$. This completes the proof that $\lambda_{*}(\infty)=0$, and also the proof of Theorem 5 .

\section{REFERENCES}

1. H. Federer, Geometric measure theory, Die Grundlehren der math. Wissenschaften, Band 153, Springer-Verlag, New York, 1969. MR 41 \#1976.

2. J.-P. Kahane, Séries de Fourier absolutment convergentes, Ergebnisse der Mathematik und ihrer Grenzgebiete, Band 50, Springer-Verlag, Berlin and New York, 1970. MR 43 \#801.

3. R. P. Kaufman, A functional method for linear sets, Israel J. Math. 5 (1967), 185187. MR $38 \# 4902$.

4. - Sets of multiplicity and differentiable functions, Proc. Amer. Math. Soc. 32 (1972), 472-476. MR 49 \#5678.

5. - Menshoff's set of mulitplicity, Math. Scand. 34 (1974). \#1169.

6. R. Salem, Algebraic numbers and Fourier analysis, Boston, Mass. 1963. MR 28

7. H. Whitney, On totally differentiable and smooth functions, Pacific J. Math. 1 (1951), 143-159. MR 13, 333.

8. A. Zygmund, Trigonometric series. Vol. II, Cambridge Univ. Press, New York, 1959, 1968. MR 21 \#6498; 38 \#4882. 